\title{
Screening of prognostic biomarkers for endometrial carcinoma based on a ceRNA network
}

\author{
Ming-Jun Zheng ${ }^{1}$, Rui Gou ${ }^{1}$ ， Wen-Chao Zhang ${ }^{1}$, Xin Nie ${ }^{1}$, Jing Wang ${ }^{1}$ ， Ling-Ling Gao ${ }^{1}$, Juan-Juan Liu ${ }^{1}$, \\ Xiao Li ${ }^{1}$, Bei Lin ${ }^{\text {Corresp. } 1}$ \\ ${ }^{1}$ Shengjing Hospital Affiliated to China Medical University, China Medical University, Liao ning province, Shen yang, China \\ Corresponding Author: Bei Lin \\ Email address: linbei88@hotmail.com
}

Objective: This study aims to reveal the regulation network of IncRNAs-miRNAs-mRNA in endometrial carcinoma (EC), to investigate the underlying mechanisms of EC occurrence and progression, to screen prognostic biomarkers. Methods: RNA-seq and miRNA-seq data of endometrial carcinoma were downloaded from the TCGA database. Edge.R package was used to screen differentially expressed genes. A database was searched to determine differentially expressed IncRNA-miRNA and miRNA-mRNA pairs, to construct the topological network of ceRNA, and to elucidate the key RNAs that are for a prognosis of survival. Results: We screened out 2632 mRNAs, 1178 IncRNAs and 189 miRNAs that were differentially expressed. The constructed ceRNA network included 97 IncRNAs, 20 miRNAs and 73 mRNAs. Analyzing network genes for associations with prognosies revealed 169 prognosis-associated RNAs, including 92 IncRNAs, 16miRNAs and 61 mRNAs.

Conclusion: Our results reveal new potential mechanisms underlying the carcinogenesis and progression of endometrial carcinoma. 
1 Screening of prognostic biomarkers for endometrial carcinoma based on

\section{2 a ceRNA network}

3 Ming-Jun Zheng ${ }^{1}$, Rui Gou ${ }^{1}$, Wen-Chao Zhang ${ }^{1}$, Xin Nie ${ }^{1}$, Jing Wang ${ }^{1}$, Ling-Ling Gao ${ }^{1}$, Juan4 Juan Liu ${ }^{1}$ Xiao $\mathrm{Li}^{1}$ and Bei Lin*

$5 \quad{ }^{1}$ Shengjing Hospital Affiliated to China Medical University, China Medical University, Liao ning 6 province, Shen yang, China

7 Corresponding author: Bei Lin

8 Email address: linbei88@hotmail.com endometrial carcinoma (EC), to investigate the underlying mechanisms of EC occurrence and progression, to screen prognostic biomarkers.

Methods: RNA-seq and miRNA-seq data of endometrial carcinoma were downloaded from the TCGA database. Edge.R package was used to screen differentially expressed genes. A database was searched to determine differentially expressed lncRNA-miRNA and miRNA-mRNA pairs, to construct the topological network of ceRNA, and to elucidate the key RNAs that are for a prognosis of survival.

Results: We screened out 2632 mRNAs, 1178 lncRNAs and 189 miRNAs that were differentially expressed. The constructed ceRNA network included 97 lncRNAs, 20 miRNAs and 73 mRNAs. Analyzing network genes for associations with prognosies revealed 169 prognosis-associated RNAs, including 92 lncRNAs, 16miRNAs and 61 mRNAs.

Conclusion: Our results reveal new potential mechanisms underlying the carcinogenesis and 24 progression of endometrial carcinoma.

\section{Introduction}

Endometrial carcinoma (EC) is the most common malignant gynecologic tumor. It is also the fourth most common malignant tumor in women following breast, lung and colorectal cancers 
29 are the main reasons for death caused by EC. It has been found that EC development is related not 30 only to hormones, but also to gene mutation. EC is mainly classified into two subtypes: estrogen 31 dependent subtype (subtype I) and gene mutation-associated subtype (subtype II). Subtype I 32 comprises $80 \%$ of new incidence, is relatively well-differentiated, and has a high five-year survival 33 rate. Subtype II (gene mutation-associated) EC is histologically poorly differentiated, highly 34 invasive and has a five-year survival rate of only 30-40\% ( Bokhman JV,1983; Jia N et al.,2014;

Matias-Guiu X, Davidson B, 2014) . It has been shown that mutations in p53, ATR and other genes correlate with EC carcinogenesis and prognosis (Li M,2014; Zighelboim I,2009). In recent years, competing endogenous RNA (ceRNA) ( Tay, Y, Rinn, J. \& Pandolfi, P. P, 2014) has become a hotspot in cancer research. MicroRNAs (miRNAs) play important roles in post-transcriptional regulation by inhibiting target gene expression; EC carcinogenesis is also associated with miRNA expression. It was revealed that miR-182 promotes EC cell proliferation by targeting the tumor suppressor gene TCEAL7 (Guo Y,2013). Sun H et al. constructed the TF-miRNA-mRNA network and found that miR-141-3p and miR-130b-3p are involved in the carcinogenesis and development of EC (Sun H et al, 2017). Long non-coding RNAs ( $\operatorname{lncRNAs} 0$ act as key players regulating gene expression during the EC transformation and progression through signaling, decoying, guiding and scaffolding at the epigenetic, transcriptional and post-transcriptional levels (Smolle M, 2015; Rinn JL, Chang HY, 2012 ; Carlson HL,2015; Martianov I, 2007; Yoon J-H, Abdelmohsen K \& Gorospe M, 2013 ; Wang KC, Chang HY, 2011) .Several lncRNAs have been found to work as prognostic indicators for cancer. Through the ceRNA regulation network in ovarian cancer, Meng Zhou et al. identified a group of prognostic biomarkers for ovarian cancer comprising 10 lncRNAs (Zhou M et al, 2016). Xu et al. utilized lncRNA-mRNA expression profiling, finding that six lncRNAs, including KIAA0087, correlated with EC prognosis (Xu, J. et al , 2016). Based on these findings, we believe that classification based on new molecular subtypes would benefit the early diagnosis and treatment of EC. 
54 Interaction mechanisms in the lncRNA-miRNA-mRNA regulation network in EC remain unclear.

55 Thus, investigating the relationships among these molecules and to find effective tumor

56 biomarkers is critical to improve patient prognoses. Therefore, we constructed a topological

57 network of ceRNA in EC and screened RNA biomarkers for prognosis, thereby identifying

58 921ncRNAs, 16 miRNAs and 61mRNAs that are related to patient prognosis and survival. Finally,

59 we defined a group of prognostic biomarkers comprising eight mRNAs.

60

61 Materials and methods:

\section{1. Data sources and pretreatment}

63 EC RNA-seq and miRNA-seq data were downloaded from the TCGA database (http://gdc64 portal.nci.nih.gov/) ( Tomczak, K., Czerwińska, P. \& Wiznerowicz, M, 2015). RNA-seq data included 65551 EC and 35 paracancerous samples. miRNA-seq data included 546 EC and 33 paracancerous samples. As the data was obtained from the TCGA website, further approval by an ethics committee was not required. This study meets the publication guidelines provided by TCGA (http://cancergenome.nih.gov/publications/publicationguidelines). RNA-seq and miRNA-seq matrix files describing samples were downloaded from TCGA. After that, mRNA and lncRNA expression profiles were extracted from the RNA-seq matrix information. Thus, we obtained three matrix files: mRNA, lncRNA and miRNA expression profiles.

\section{Screening of differentially expressed genes}

Statistical analysis was performed using the exactTest function in the edge.R software package (Robinson, M. D., McCarthy, D. J. \& Smyth, G. K, 2010). and differentially expressed genes were screened out. Next, the P value was FDR-corrected. mRNA, lncRNA and miRNA with FDR $<0.05$ were screened out. RNAs with fold change $>4(|\log \mathrm{FC}|>2)$ were further selected as differentially generated. 


\section{3. ceRNA topological network construction}

\section{$82 \quad 3.1$ IncRNA-miRNA pair screening}

83 MiRcode (Jeggari, A., Marks, D. S. \& Larsson, E, 2012) is database that predicts miRNA targets based 84 on a transcriptome annotated by human GENCODE. Through miRcode matching, we determined 85 the miRNAs that interacted with differentially expressed lncRNAs and compared them to the pairing files.

\subsection{Screening of miRNA-mRNA pairs}

MiRDB (Wong, N. \& Wang, X, 2015) is a database that can be used to predict miRNA target genes based on high throughput sequencing data. miRTarBase (Chou CH,2016) is an experimentallyverified miRNA target gene database. TargetScan (Lewis, B. P., Burge, C. B. \& Bartel, D. P, 2005) predicts miRNA targets by searching for the presence of conserved 8mer and 7 mer sites that match the seed region of an input miRNA. We predicted target genes of the miRNA in the lncRNAmiRNA pairs through these three aforementioned databases, and defined target genes that were predicted by all three databases (miRDB, miRTarBase and TargetScan) as the final screened-out target genes. These genes were compared to the differentially expressed genes mentioned above, and genes in common were selected to build an miRNA-mRNA pairing file. Finally, a ceRNA topological network was constructed based on the abovementioned pairing files.

\section{Topological and stability analysis of the ceRNA network}

103 The NetWorkAnalyzer toolkit (Shannon P, 2003) from Cytoscape (Doncheva, N. T., 2012) was used

104 to analyze the characteristics of the ceRNA topological network. NetWorkAnalyzer is mainly used to analyze network diameter, connection numbers and average clustering coefficient. In this study, we calculated network connections, the path length and the closest centrality of nodes. 


\section{Functional and enrichment analysis of ceRNA}

109 Biological function characterization of DEmRNAs in the ceRNA network was performed using

110 the Database for Annotation, Visualization, and Integrated Discovery (DAVID) version 6.7

111 (https://david-d.ncifcrf.gov/home.jsp), and pathway enrichment analysis was performed using the

112 KO-Based Annotation System (KOBAS) 3.0 (http://kobas.cbi.pku.edu.cn/)( Huang da W,

113 Sherman BT \& Lempicki RA. 2009; Xie C, Mao X, Huang J, et al, 2011)

114

115 6. Prognostic analysis of the ceRNA module

116 Clinical information of samples was downloaded from the TCGA database, and survival data

117 were extracted. Combined with expression profile data, a Kaplan-Meier (K-M) survival curve

118 was generated for each node in the ceRNA topological network using survival (Therneau, T. M.,

119 \& April,1994) in the R package. Survival differences for genes, lncRNAs and miRNAs in the

120 ceRNA module were also analyzed. Patients were dichotomized for survival analysis using a log-

121 rank test with optimal cutoff values determined by the "surv_cutpoint" function of the

122 “survminer” R package. $\mathrm{P}<0.05$ was considered statistically significant.

\section{Validation of DEmRNAs and DEmiRNAs in the ceRNA network}

124 The GSE17025(Allard J, et al, 2008) and GSE35794 datasets were used to validate the expression

125 of candidate mRNAs and miRNAs, respectively. Transcriptome profiling data in the GSE17025

126 (Platform: GPL570) dataset contained 91 EC and 12 non-tumor samples. The microRNA 
expression profile in the GSE35794 (Platform: GPL10850) dataset contained 18 EEC and 4

128

129

130

131

132

133

134

135

136

137

138

139

140

141

142

143

144

145

146

147

148

149

150

151

152

153

normal samples.

\section{Results}

\section{Screening of differentially expressed RNAs}

The mRNA expression profiles of 35 normal samples and 551 EC samples were compared. After statistical validation, 2632 differentially expressed mRNAs were screened out including 1672 upregulated and 960 downregulated mRNAs (Fig. 1 A,D; Table S1). We obtained 1178 differentially expressed lncRNAs after screening, including 867 that were upregulated and 311 that were downregulated. Results are shown in Fig. 1 B,E; Table S1.

The miRNA expression profiles of 33 normal samples and 546 EC samples were compared, and 189 differentially expressed miRNAs were obtained after statistical validation. One hundred and forty of these 189 miRNAs were upregulated and 49 were downregulated. Results are shown in Fig. 1C,F; Table S1.

\section{Construction of the ceRNA network and analysis of topology and stability}

Based on the differentially expressed lncRNA and miRNA profiles, lncRNA and corresponding miRNAs were paired using the miRcode database. We established 556 pairs, with 99 lncRNAs and 27 miRNAs (Table S2). Twenty-five of these 27 DEmiRNAs were found in the starBase database. The target genes of these 25 miRNAs were predicted using three public databases: miRDB, miRTarBase and TargetScan. Nine hundred and fifty-six target genes were found in total. These target genes were compared with 2632 differentially expressed genes that were screened out, and genes in common were selected out. Seventy-three DEmRNAs that could interact with 20 of the 25 DEmiRNAs in all three datasets were selected (Table S3). After removing the remaining seven DEmiRNAs and corresponding lncRNAs, 97 DElncRNAs, 20 DEmiRNAs and 73 
154 DEmRNAs were ultimately used to construct a ceRNA network used the "ggalluvial" R package

155 for visualization (Fig. 2A; Table S4).

156 The topology of the ceRNA network was analyzed to verify the network's reliability. The

157 distribution of the numbers of nodes is shown in Fig. 2B. Node number decreased with increases

158 in distribution degree. The highest degree was 51; the lowest was zero. Most nodes in the network

159 were nodes with fewer interactions. The closeness centrality (CC) of a given node can be used

160 calculate the connection steps from the node to another. More centralized nodes show higher

161 scores. Closeness centrality indicates the shortest path, and we demonstrate in Fig. 2C that there

162 were more nodes displaying the same numbers of connections, and that these nodes are relatively

163 centralized in the network. Those nodes with more connections are relatively sparsely distributed.

164 Path reflects the combination of all nodes in the network. Fig. 2D shows the shortest pathway

165 length distribution in the network, demonstrating that path length is mainly focused in the center.

166 There were fewer extreme values in this analysis. The upper threshold was four and the lower

167 threshold was one, suggesting that the majority of nodes in the network can be connected by a

168 relatively short path.

169 Node degree distribution density is shown in Fig. 2E. We found that the number of nodes

170 decreased as node degree increased, indicating that most nodes in the network are isolated. It is

171 possible that during diseases occurrence, some key nodes change and interact with nearby nodes;

172 therefore, co-expression occur and downstream biological processes are affected. In addition, we

173 calculated the connection degree of each gene by topology to clarify its importance in the ceRNA

174 network (Table S5). MEG3 (connection degree $=21)$, hsa-mir-195 $($ connection degree $=51)$ and

175 ZEB1 (connection degree $=6$ ) were considered the most important genes among the lncRNAs,

176 miRNAs, and mRNAs, respectively. Because hsa-mir-195 was the gene with the highest

177 connection degree in the ceRNA network, we conclude that it might exert a strong influence on

178 EC tumorigenesis.

179 


\section{Positive correlations among the ceRNA expression levels}

181 According to ceRNA theory, lncRNAs can positively regulate mRNAs by competitively

182 competing with miRNAs (Fig. 2A). To verify this finding, regression analysis was performed

183 between the DElncRNAs and DEmRNAs targeted by hsa-mir-195. Strong positive correlations

184 were found between the DElncRNAs and DEmRNAs targeted by hsa-miR-195 (minimum

185 requirement of an interaction score $>0.3$; Fig. 3; Table S6). Fig. 3 A-F shows the top six

186 interactions in which C2orf48 interacts with PSAT1, C2orf48 interacts with KIF23, C2orf48

187 interacts with CCNE1, C2orf48 interacts with CEP55, C2orf48 interacts with CBX2, and

188 C2orf48 interacts with CDC25A under hsa-mir-195 regulation.

189

190

191 4. Biological processes and pathway enrichment analysis of the ceRNA network

192 Seventy-three mRNAs in the ceRNA network were subjected to Gene Ontology (GO) and

193 pathway enrichment analysis using the DAVID 6.7 database. The first 20 biological functions

194 that demonstrated statistical significance were analyzed by "GOplot”.R package. Pathways were

195 visualized with Cytoscape software. GO analysis demonstrated that the biological processes

196 significantly enriched by mRNAs in the ceRNA network include GO:0000187 activation of

197 MAPK activity, GO:0060412 ventricular septum morphogenesis and GO:0045599 negative

198 regulation of fat cell differentiation (Fig. 4A; Table S7). Kyoto Encyclopedia of Genes and

199 Genomes (KEGG) pathway enrichment analysis demonstrated that these genes are involved in

200 regulating microRNAs in cancer, p53 signaling pathways, the cell cycle, the Rap1 signaling 
201 pathway and other signaling pathways closely associated with tumorigenesis (Fig. 4B; Table 202 S8).

\section{Survival and gene expression analysis}

205

To clarify how DElncRNAs, DEmiRNAs, and DEmRNAs in the ceRNA network affect the prognoses of patients suffering from EC, K-M survival analysis was performed for the 97 lncRNAs, 20 miRNAs and 73 mRNAs, separately. The results demonstrate that 92 of the 99 lncRNAs were significantly associated with prognosis based on their respective optimal cutoffs (Kassambara A et al, 2014) and 59 DElncRNAs exhibited positive correlations with overall

211 survival; in contrast, the remaining 33 DElncRNAs exhibited negative associations with overall

212 survival (log-rank P $<0.05$; Table S9). Sixteen of the 20 differentially expressed miRNAs

213 correlated to prognosis. Ten DEmiRNAs positively correlated to overall survival, and the

214 remaining 6 DEmiRNAs were negatively associated with overall survival (log-rank $\mathrm{P}<0.05$;

215 Table S10). Sixty-one of the 73 DEmRNAs were highly relevant for overall survival based on

216 their respective optimal cutoffs. Forty-four DEmRNAs positively correlated to overall survival,

217 and the remaining 17 DEmRNAs were negatively correlated to overall survival (log-rank P <

218 0.05; Table S11). Fig. 5A-D shows the survival curves of the top four DElncRNAs (FAM41C,

219 ADARB2.AS1, C8orf49 and MIR7.3HG), Fig. 5 E-H shows the survival curves of the top four

220 DEmiRNAs (has-mir-195, has-mir-140, has-mir-205 and has-mir-200a) and Fig. 5I-L shows the 221 survival curves of the top four DEmRNAs (PSAT1, KIF23, MCM4 and CCNE1). Then, we

222 verified the expression of four mRNAs and miRNAs through the TCGA endometrial cancer 223 dataset, and found that hsa-mir-195 and hsa-mir-140 are poorly expressed in endometrial 224 cancer(Fig. 5 Q-R). The survival curves showed that as expression decreases, patient prognoses 225 worsen, suggesting that these two miRNAs promote endometrial cancer development by 
226 negatively regulating the expression of cancer-promoting genes. Hsa-mir-205 and hsa-mir-200a

227 were highly expressed in endometrial carcinoma tissues(Fig. 5 S-T), and their survival curves

228 showed that as gene expression increases, survival prognosis worsens. We conclude that both

229 miRNAs negatively regulate the expression of tumor suppressor genes and thereby promote the

230 occurrence and development of tumors. The four genes that are highly expressed in endometrial

231 cancer tissues (PSAT1, KIF23, CCNE1 and MCM4) showed a pattern whereby increasing

232 expression was associated with worsening five-year survival prognosis（Fig. 5 M-P） .

233

234 6. Analysis of correlations between gene expression and clinicopathological parameters

235 The UALCA online database (Chandrashekar, D. S. et al ,2017) was used to analyze correlations

236 between mRNA expression of the above genes and patient clinicopathological parameters. We

237 found that PSAT1, KIF23, CCNE1 and MCM4 expression was higher at different clinical stages

238 than in the control group, and expression was higher in different pathological subtypes than in the

239 normal group. At the same time, we found that the expression levels of all four genes was higher

240 in the serous subtype than in endometroid type. All differences were statistically significant,

241 suggesting that these four markers could also be used as biomarkers for molecular subtypes of EC

242 (Fig. 6 A-H).

243

\section{Validation of DEmRNAs and DEmiRNAs in the ceRNA network}

The top four DEmRNAs and DEmiRNAs correlated with overall survival were selected for

validation. Consistent with our earlier results, mean expression levels of all four mRNAs were

significantly higher in EC tissues than in non-cancerous tissues in the GSE17025 dataset (Fig.

7A-D). Mean expression levels of hsa-mir-195, hsa-mir-140 were significantly lower in EC 
250

251

252

253

expression was significantly higher in EC tissues. However, due to the small number of patients, no significant differences were found in has-mir-200a expression (Fig. 7 G-H).

\section{Flow chart of construction and analysis of ceRNA network}

The flow chart of construction of lncRNAs-miRNAs-mRNA regulation network in endometrial carcinoma of is shown in Fig.8

\section{Discussion}

Endometrial cancer (EC) is the most common gynecological tumor in women. Its carcinogenesis and progression are driven by multiple mechanisms, many of which interact with one another. Therefore, it is important for early diagnosis and treatment biomarker discovery to investigate the underlying mechanisms of EC occurrence and development, and to screen prognosis-associated RNAs.

A growing number of investigations have confirmed that lncRNAs act as key players regulating gene expression during the EC transformation and progression through signaling, decoying, guiding and scaffolding at the epigenetic, transcriptional and post-transcriptional levels. Many studies have indicated that a complicated regulatory network exists between lncRNAs and miRNAs. MicroRNAs (miRNAs) play important roles in post-transcriptional regulation by inhibiting target gene expression; EC carcinogenesis is also associated with miRNA expression. Therefore, these RNAs could be used as cancer-related prognostic biomarkers.

In the present study, we constructed a ceRNA regulation network composed of 97 lncRNAs, 20 miRNAs and 73 mRNAs. Among these RNAs, the expression levels of 92 lncRNAs, 16miRNAs and 61 mRNAs were correlated to prognostic survival. 
273 Genes related to overall survival are likely to reflect on the prognosis of EC.

274 IncRNAs are non-coding protein RNA molecules of more than 200 nucleotides in length, and

275 recent studies have reported an increasing number of functional lncRNAs. Maternal expressed

276 gene 3 (MEG3) is a maternal imprinted gene encoding a lncRNA that acts as a tumor suppressor

277 in various tumors. In the constructed ceRNA topology network, lncRNA MEG3 showed the

278 highest degree of connectivity of included DElncRNAs (degree of connectivity $=21$ ), and its

279 expression is downregulated in EC tissues. Sun et al. (Sun, K. X.et al,2017) found that MEG3

280 expression in EC tissues is significantly lower than in normal endometrial tissues. MEG3

281 overexpression inhibits proliferation, invasion and metastasis of EC cells and promotes apoptosis

282 by inhibiting activation of the phosphoinositide 3-kinase (PI3K)/m-TOR signaling pathway. We

283 also found that MEG3 is associated with 16 key DEmiRNAs (hsa-mir-429, hsa-mir-205, hsa-mir-

284 141, hsa-mir-204, hsa-mir-182, hsa-mir-424, hsa-mir-140, hsa-mir-143, hsa-mir-216b, hsa-mir-

285 106a, hsa-mir-96, hsa-mir-145, hsa-mir-200a, hsa-mir-122, hsa-mir- 211 and hsa-mir-195) and

286 competes to regulate mRNA expression in EC. Of these, hsa-mir-195 is the highest of all nodes,

287 and interacts with most of the DElncRNAs and DEmRNAs in the ceRNA network, suggesting that

288 hsa-mir-195 has a significant effect on EC pathogenesis and prognosis.Yang et al.( Yang Y et al,

289 2014) found that miR-195 is down-regulated in hepatocellular carcinoma tissues and cells.

290 Overexpression of miR-195 can target Wnt3a, inhibit proliferation of hepatocellular carcinoma

291 cells, and promote apoptosis. In our study, we found that miR195 is also significantly

292 underexpressed in endometrial cancer tissues, and the survival curve suggests that its low

293 expression is associated with poor prognosis. This indicates that miR195 inhibits the expression

294 of oncogenes and promotes the development of endometrial cancer.

295 Our regression analysis of IncRNAs and mRNAs associated with miR195 revealed a significant 296 correlation between C2orf48, PSAT1, KIF23, CCNE1, CDC25A and CBX2. Among these,

297 C2orf48 had the strongest correlation with KIF23 (Person's $\mathrm{r}=0.617 ; \mathrm{p}<0.001$ ), and C2orf48, 298 PSAT1, KIF23, CCNE1, CDC25A, and CBX2 were significantly upregulated in EC tissues as 
compared to adjacent tissues. Survival analysis showed that the higher the expression levels of IncRNAs C2orf48, PSAT1, KIF23, CCNE1, CDC25A and CBX2 in patients with endometrial cancer, the poorer the prognosis. Furuta et al.( Furuta M, 2013) successfully demonstrated that miR195 can inhibit CDC25A and CCNE1 expression thereby regulating the cell cycle and

303 inducing hepatoma cell proliferation.

304 To validate the expression of prognostic-related molecules in the constructed ceRNA network, we 305 selected four mRNAs (PSAT1, KIF23, MCM4 and CCNE1) and miRNAs (mir-195, mir-140, mir306205 and mir-200a ) that are the most relevant to prognosis in the ceRNA network, and verified in 307 the mRNA and miRNA expression profiles in an external dataset of endometrial carcinoma from 308 GEO. The results were consistent with those of the TCGA database. There was no significant 309 difference in the differential expression of mir-200a due to the small number of external samples.

310 The above results demonstrate to some extent the reliability of the ceRNA network constructed by 311 prognosis-related RNA after screening. At the same time, we used the TCGA database to analyze 312 correlations between PSAT1, KIF23, CCNE1, MCM4 and clinical pathological parameters, 313 finding that their expression levels in serous endometrial cancer are significantly higher than in 314 endometriod endometrial carcinoma, suggesting that these four RNAS could also be used as 315 molecular biomarkers of EC.

316 The KIF23 gene belongs to the KIF family and has been found to play an important role in the

317 process of mitotic cytoplasmic separation. KIF23 overexpression is significantly associated with

318 tumor grade, invasion and prognosis in breast cancer (Zou JX et al., 2014). High expression of 319 KIF23 in glioma cells may be related to transcriptional activation, and in vivo and in vitro 320 experiments have demonstrated.

321 KIF23 knockdown significantly inhibits glioma cell proliferation (Takahashi, S.,2012)

322 And it was also found to be abnormally expressed in gastric cancer, NSCLC tissues (Murakami H 323 et al., 2013 ; Kato T et al., 2016). 
324 However, there is no research report on KIF23 in EC. Our study found that it is highly expressed

325 in EC, is regulated by mir-195 is associated with poor prognosis, and can also be used as a marker

326 for a subtype of EC.

327 PSAT1 is an enzyme involved in serine synthesis. PSAT1 overexpression correlates to poor 328 prognosis in colon cancer, non-small cell lung cancer and breast cancer.(Martens JW et al., 2005;

329 Pollari, S.et al, 2012; Vié, N. et al, 2008; Yang Y et al, 2015). To date, no report has focused on PSAT1

330 in EC. Yan et al.( Yan S et al, 2015) demonstrated that miRNA-340 inhibits esophageal cancer cell

331 proliferation and invasion. PSAT1 overexpression is a marker for poor prognosis in

332 nasopharyngeal carcinoma, as well.( Liao KM et al., 2016) .Based on our research, we believe that

333 lncRNA C2orf48 can mediate PSAT1 expression by competing with miR-195, thereby promoting

334 EC progression.

335 Conclusion

336 To summarize, we constructed a ceRNA topological network based on RNA-seq and miRNA-seq

337 data of EC in the TCGA public database, analyzed correlations of nodes in the network to patient

338 survival, and identified multiple RNAs that possibly affect prognosis at the transcriptional level.

339 We describe a C2orf48-hsa-mir-195 ceRNA functional model associated with EC development

340 and prognosis, and verified prognostic RNA expression using external data.

341 Finally, it should be noted that we did not conduct clinical trials to verify the relationship between

342 the predicted RNA molecules and EC. Clinical studies require appropriate sample sizes and

343 longitudinal designs to verify the results: the purpose of this study is to identify potential

344 prognostic value of lncRNAs, miRNAs and mRNAs by constructing ceRNA networks to serve as

345 a basis for further experimental and clinical research. In addition, the data on normal and cancer

346 samples in the TCGA database are not matched, and therefore our research results also need further

347 validation via sample as well as in vitro and in vivo experiments.

348

349 
351 Table S1. Differentially expressed genes between EC samples and adjacent non-tumor samples.

352 Table S2. The 99 DEIncRNAs interacting with the 27 DEmiRNAs retrieved from the miRcode database.

353 Table S3. The 20 DEmiRNAs interacting with the 73 DEmRNAs retrieved from the miRDB,

354 miRTarBase and TargetScan databases.

355 Table S4. Interactions of the ceRNA network in endometrial carcinoma.

356 Table S5. The connection degree of each gene in the ceRNA network.

357 Table S6. Linear regression analysis between the DEIncRNAs and DEmRNAs targeted by hsa-mir-195.

358 Table S7. Functional enrichment analysis of the 73 DEmRNAs.

359 Table S8. Pathway enrichment analysis of the 73 DEmRNAs.

360 Table S9.92 DEIncRNAs associated with overall survival in endometrial carcinoma.

361 Table S10. 16 DEmiRNAs associated with overall survival in endometrial carcinoma.

362 Table S11. 61 DEmRNAs associated with overall survival in endometrial carcinoma.

[1] Siegel RL, Miller KD, Jemal A. 2017.Cancer Statistics. CA: a cancer journal for clinicians. 67(1) : 730.

368 [2] Bokhman JV. 1983. Two pathogenetic types of endometrial carcinoma. Gynecol Oncol .15(1): 10-17. 
369 [3] Jia N, Li Q, Tao X, Wang J, Hua K, Feng W. 2014. Enhancer of zeste homolog 2 is involved in the

370

371

372

373

374

375

376

377

378

379

380

381

382

383

384

385

386

387

388

389

390

391

392

393

394

395

396

397

398

399

400

401

402

403

404

405

406

407 proliferation of endometrial carcinoma. Oncol Lett. 8: 2049-2054.

[4] Matias-Guiu X, Davidson B. 2014. Prognostic biomarkers in endometrial and ovarian carcinoma. Virchows Arch. 464: 315-331.

[5] Li M, Zhao L, Shen D, Li X, Wang J, Wei L. 2014. Clinical implications and prognostic value of single and combined biomarkers in endometrial carcinoma. Chin Med J (Engl) . 127: 1459-1463.

[6] Zighelboim I, Schmidt AP, Gao F, Thaker PH, Powell MA, Rader JS, Gibb RK, Mutch DG and Goodfellow PJ. 2009. ATR mutation in endometrioid endometrial cancer is associated with poor clinical outcomes. $\mathrm{J}$ Clin Oncol, 27(19): 3091-3096.

[7] Guo Y, Liao Y, Jia C, Ren J, Wang J, Li T. 2013. MicroRNA-182 promotes tumor cell growth by targeting transcription elongation factor Alike 7 in endometrial carcinoma. Cell Physiol Biochem. 32: 581-590.

[8] Tay, Y, Rinn, J. \& Pandolfi, P. P. 2014. The multilayered complexity of ceRNA crosstalk and competition. Nature, 505(7483): 344-352.

[9] Sun H, Yan L, Tu R, Zhang Y, Ma L, Tang W, Li L, Chen W, Zhan C, Zang R. 2017. Expression Profiles of Endometrial Carcinoma by Integrative Analysis of TCGA Data. Gynecol Obstet Invest . 82: 30-38

[10] Smolle M, Bullock M, Ling H, Pichler M, Haybaeck J. 2015. Long Non-Coding RNAs in Endometrial Carcinoma. International Journal of Molecular Sciences . 16(11): 26463-26472

[11] Rinn JL, Chang HY. 2012. Genome regulation by long noncoding RNAs. Annual review of biochemistry. 81: 145-166.

[12] Carlson HL, Quinn JJ, Yang YW, Thornburg CK, Chang HY, Stadler HS. 2015. LncRNA-HIT Functions as an Epigenetic Regulator of Chondrogenesis through Its Recruitment of p100/CBP Complexes. PLoS genetics .11(12): e1005680

[13] Martianov I, Ramadass A, Serra Barros A, Chow N, Akoulitchev A. 2007. Repression of the human dihydrofolate reductase gene by a non-coding interfering transcript. Nature . 445(7128): 666-670.

[14] Yoon J-H, Abdelmohsen K, Gorospe M. 2013. Posttranscriptional Gene Regulation by Long Noncoding RNA. Journal of Molecular Biology . 425(19): 3723-3730.

[15] Wang KC, Chang HY. 2011. Molecular mechanisms of long noncoding RNAs. Molecular cell .43(6): 904914.

[16] Meng, Z., Wang, X., Shi, H., Liang, C., Wang, Z., Zhao, H., Yang L.,Sun J. 2016. Characterization of long non-coding rna-associated cerna network to reveal potential prognostic lncrna biomarkers in human ovarian cancer. Oncotarget, 7(11), 12598-12611.

[17] Xu, J., Qian, Y., Ye, M., Fu, Z., Jia, X., Li, W., Xu, P., Lv, M., Huang, L., Wang, L., Ruan, H., Lv, J. 2016. Distinct expression profile of IncRNA in endometrial carcinoma. Oncology Reports. 36(6): 34053412.

[18] Tomczak, K., Czerwińska, P. \& Wiznerowicz, M. 2015. The Cancer Genome Atlas (TCGA): an immeasurable source of knowledge. Contemporary Oncology . 19(1A): A68-77.

[19] Robinson, M. D., McCarthy, D. J. \& Smyth, G. K. 2010. edgeR: a Bioconductor package for differential expression analysis of digital gene expression data. Bioinformatics. 26(1): 139-40.

[20] Jeggari, A., Marks, D. S. \& Larsson, E. 2012. miRcode: a map of putative microRNA target sites in the 
long non-coding transcriptome. Bioinformatics. 28(15):2062-2063

[21] Wong, N. \& Wang, X. 2015. miRDB: an online resource for microRNA target prediction and functional annotations. Nucleic Acids Research. 43(Database issue): D146-52.

[22] Chou CH, Chang NW, Shrestha S, Hsu SD, Lin YL, Lee WH, Yang CD, Hong HC, Wei TY, Tu SJ, Tsai TR, Ho SY, Jian TY, Wu HY, Chen PR, Lin NC, Huang HT, Yang TL, Pai CY, Tai CS, Chen WL, Huang CY, Liu CC, Weng SL, Liao KW, Hsu WL, Huang HD. 2016. miRTarBase 2016: updates to the experimentally validated miRNA-target interactions database. Nucleic Acids Research . 44(D1): D239-247.

[23] Lewis, B. P., Burge, C. B. \& Bartel, D. P. 2005. Conserved Seed Pairing, Often Flanked by Adenosines, Indicates that Thousands of Human Genes are MicroRNA Targets. Cell . 120(1): 15-20.

[24] Shannon P, Markiel A, Ozier O, Baliga NS, Wang JT, Ramage D, Amin N, Schwikowski B, Ideker T. 2003. Cytoscape: A Software Environment for Integrated Models of Biomolecular Interaction Networks. Genome Research. 13(11): 2498-2504.

[25] Doncheva, N. T., Assenov, Y., Domingues, F. S. \& Albrecht, M. 2012. Topological analysis and interactive visualization of biological networks and protein structures. Nature Protocols . 7(4): 670-85.

[26] Huang da W, Sherman BT, Lempicki RA. 2009. Systematic and integrative analysis of large gene lists using DAVID bioinformatics resources. Nature protocols. 4(1):44-57.

[27] Xie C, Mao X, Huang J, Ding Y, Wu J, Dong S, Kong L, Gao G, Li CY, Wei L.2011. KOBAS 2.0: a web server for annotation and identification of enriched pathways and diseases. Nucleic acids research. 39(Web Server issue):W316-322.

[28] Therneau, T. M., \& April. 1994. A package for survival analysis in s.

[29] Allard, J., Risinger, J. I., Day, R., Chandran, U., Litzi, T., Rose, G., A. Berchuck., George Larry Maxwell. 2008. Microarray analysis of microdissected stage i endometrial cancers reveals gene expression patterns associated with inflammation. Gynecologic Oncology, 108(3), S80-S81.

[30] Alboukadel Kassambara, Claire Gourzones-Dmitriev, Surinder Sahota, Thierry Rème, Jérôme Moreaux, Hartmut Goldschmidt, Angelos Constantinou, Philippe Pasero, Dirk Hose4, Bernard Klein.2014. A DNA repair pathway score predicts survival in human multiple myeloma: the potential for therapeutic strategy[J]. Oncotarget, 5(9):2487-98.

[31] Chandrashekar, D. S., Bashel, B., Balasubramanya, S. A. H., Creighton, C. J., Ponce-Rodriguez, I., Chakravarthi, B. V. S. K., \& Varambally, S. 2017. UALCAN: A Portal for Facilitating Tumor Subgroup Gene Expression and Survival Analyses. Neoplasia (New York, N.Y.), 19(8), 649-658. http://doi.org/10.1016/j.neo.2017.05.002

[32] Sun, K. X., Wu, D. D., Chen, S., Yang, Z., \& Zong, Z. H. 2017. Lncrna meg3 inhibit endometrial carcinoma tumorigenesis and progression through pi3k pathway. Apoptosis An International Journal on Programmed Cell Death, 22(12), 1543-1552.

[33] Yang Y, Li M, Chang S, Wang L, Song T, Gao L, Hu L, Li Z, Liu L, Yao J, Huang C. 2014. MicroRNA195 acts as a tumor suppressor by directly targeting Wnt3a in HepG2 hepatocellular carcinoma cells[J]. Molecular Medicine Reports, 10(5): 2643-2648.

[34] Furuta M, Kozaki K, Tanimoto K, Tanaka S, Arii S, Shimamura T, Niida A, Miyano S, Inazawa J. 2013. The tumor-suppressive miR-497-195 cluster targets multiple cell-cycle regulators in hepatocellular 
447

448

449

450

451

452

453

454

455

456

457

458

459

460

461

462

463

464

465

466

467

468

469

470

471

472

473

474

475

476

477

478

479

480

481

482

carcinoma. [J]. Plos One, 8(3) : e60155

[35] June X. Zou, Zhijian Duan, Junjian Wang, Alex Sokolov, Jianzhen Xu,Christopher Z. Chen, Jian Jian Li, Hong-Wu Chen. 2014. Kinesin family deregulation coordinated by bromodomain protein ANCCA and histone methyltransferase MLL for breast cancer cell growth, survival, and tamoxifen resistance. Mol Cancer Res, 12 (4): 539-549.

[36] Takahashi S, Fusaki N, Ohta S, Iwahori Y, Iizuka Y, Inagawa K, Kawakami Y, Yoshida K, Toda M. 2012. Downregulation of kif23 suppresses glioma proliferation. Journal of Neuro-Oncology, 106(3), 519-529.

[37] Murakami H, Ito S, Tanaka H, Kondo E, Kodera Y, Nakanishi H. 2013. Establishment of new intraperitoneal paclitaxel-resistant gastric cancer cell lines and comprehensive gene expression analysis. Anticancer Res, 33(10): 4299-4307.

[38] Kato T, Wada H, Patel P, Hu HP, Lee D, Ujiie H, Hirohashi K, Nakajima T, Sato M, Kaji M, Kaga K, Matsui Y, Tsao MS, Yasufuku K. 2016. Overexpression of KIF23 predicts clinical outcome in primary lung cancer patients. Lung Cancer, 92: 53-61.

[39] Martens JW, Nimmrich I, Koenig T, Look MP, Harbeck N, Model F, Kluth A, Bolt-de Vries J, Sieuwerts AM, Portengen H, Meijer-Van Gelder ME, Piepenbrock C, Olek A, Höfler H, Kiechle M, Klijn JG, Schmitt M, Maier S, Foekens JA. 2005. Association of DNA methylation of phosphoserine aminotransferase with response to endocrine therapy in patients with recurrent breast cancer.[J]. Cancer Research, 65(10): 4101-17.

[40]Vié N, Copois V, Bascoul-Mollevi C, Denis V, Bec N, Robert B, Fraslon C, Conseiller E, Molina F, Larroque C, Martineau P, Del Rio M, Gongora C. 2008 . Overexpression of phosphoserine aminotransferase psat1 stimulates cell growth and increases chemoresistance of colon cancer cells. Molecular Cancer,7,1(2008-01-25), 7(1), 14.

[41]Pollari S, Käkönen SM, Edgren H, Wolf M, Kohonen P, Sara H, Guise T, Nees M, Kallioniemi O. 2012. Enhanced serine production by bone metastatic breast cancer cells stimulates osteoclastogenesis. Breast Cancer Research \& Treatment, 131(3), 1085-1086.

[42] Yang Y, Wu J, Cai J, He Z, Yuan J, Zhu X, Li Y, Li M, Guan H. 2015. PSAT1 regulates cyclin D1 degradation and sustains proliferation of non-small cell lung cancer cells[J]. International Journal of Cancer, 136(4) : E39-E50.

[43] Yan S, Jiang H, Fang S, Yin F, Wang Z, Jia Y, Sun X, Wu S, Jiang T, Mao A. 2015. MicroRNA-340 Inhibits Esophageal Cancer Cell Growth and Invasion by Targeting Phosphoserine Aminotransferase 1[J]. Cellular Physiology \& Biochemistry International Journal of Experimental Cellular Physiology Biochemistry \& Pharmacology. 37(1): 375-386.

[44] Liao KM, Chao TB, Tian YF, Lin CY, Lee SW, Chuang HY, Chan TC, Chen TJ, Hsing CH, Sheu MJ, Li CF. 2016. Overexpression of the PSAT1 Gene in Nasopharyngeal Carcinoma Is an Indicator of Poor Prognosis[J]. J Cancer, 7(9): 1088-1094. 


\section{Figure 1}

Heatmap and volcano plots of differentially expressed RNAs.

(A) Heatmap plots of differentially expressed mRNAs. (B) Heatmap plots of differentially expressed IncRNAs. (C) Heatmap plots of differentially expressed miRNAs. The horizontal axis represents samples. Sample clusters are presented above the horizontal axis. The vertical axis represents RNAs. Red denotes upregulated genes, and green denotes downregulated genes. (D) Volcano plots of differentially expressed mRNAs. (E) Volcano plots of differentially expressed IncRNAs. (F) Volcano plots of differentially expressed miRNAs. The Y-axis denotes the log of FC (base 2 ) and the X-axis plots the negative log of the false discovery rate (FDR; base 10). Each point represents a gene. Green dots represent downregulated RNAs, red dots represent upregulated RNAs, and black dots represent non-DEGs. 
A
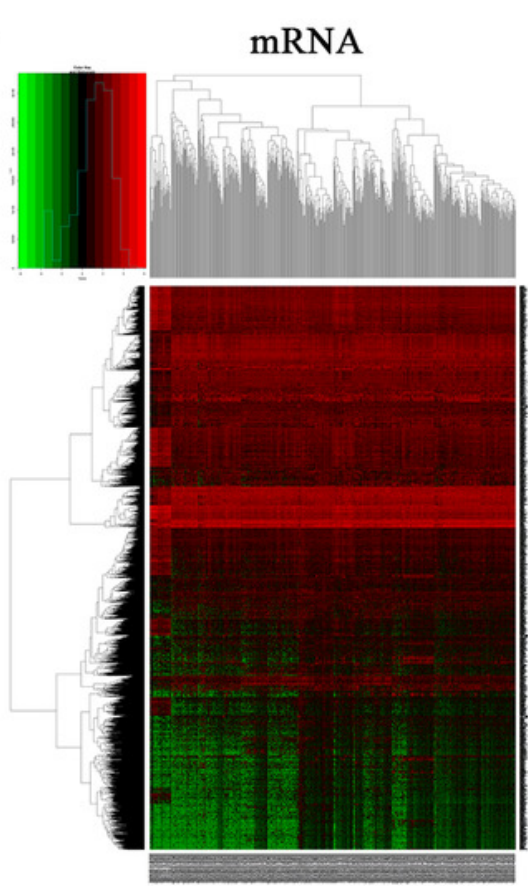

D

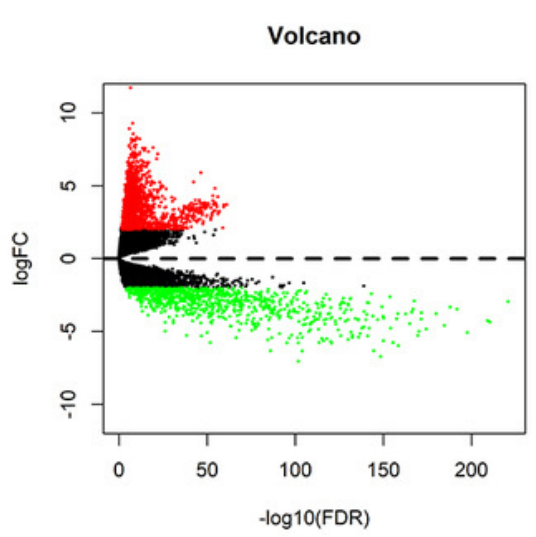

B
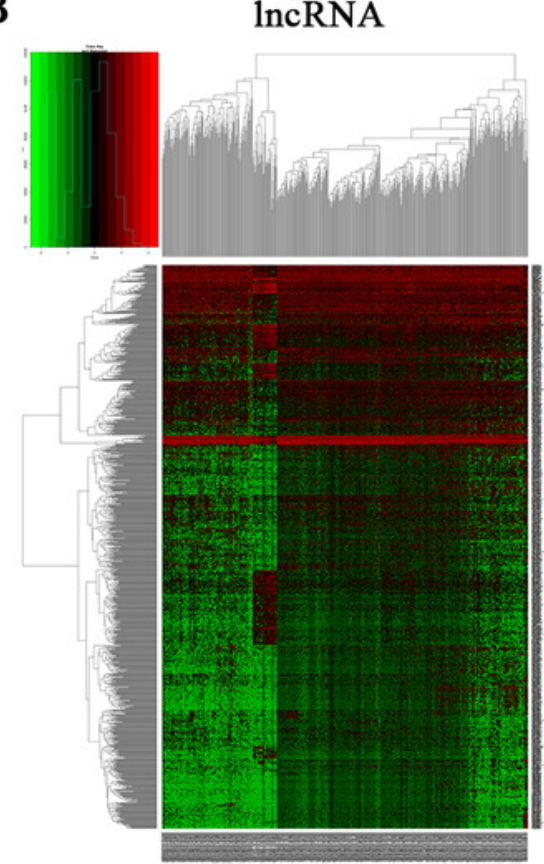

E

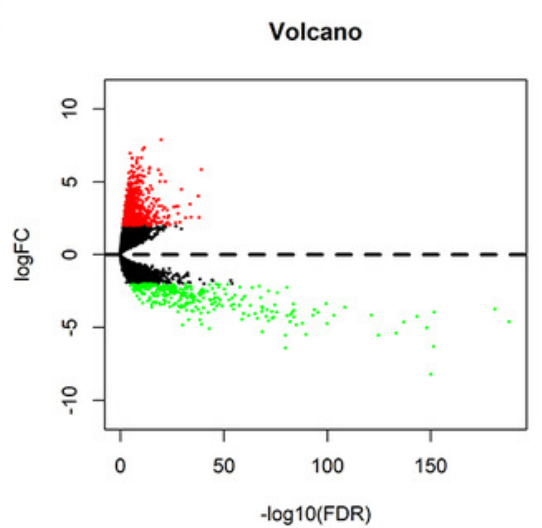

C

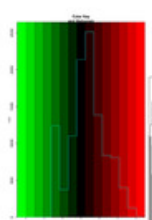

miRNA

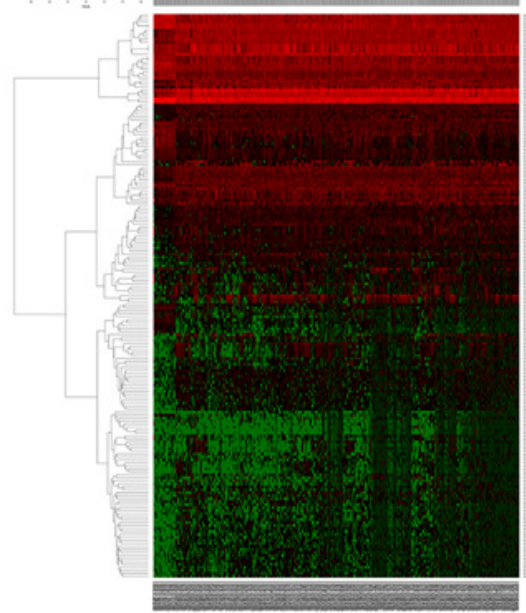

F

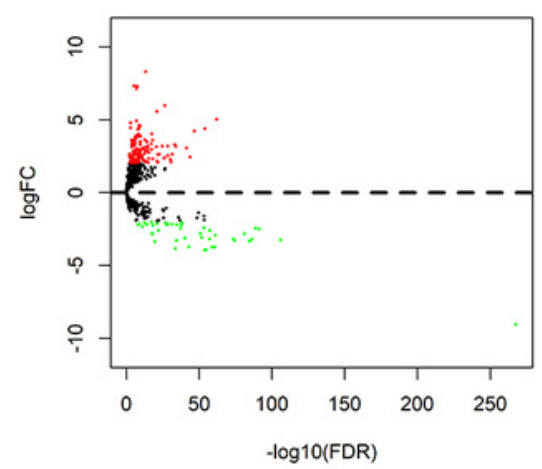




\section{Figure 2}

Sankey diagram for the ceRNA network in EC and topology, and stability analysis.

(A) Each rectangle represents a gene, and the connection degree of each gene is displayed based on the size of the rectangle. (B) Node degree distribution analysis. (C) Closeness centrality distribution. (D) Shortest path distribution. (E) Node degree distribution density. 

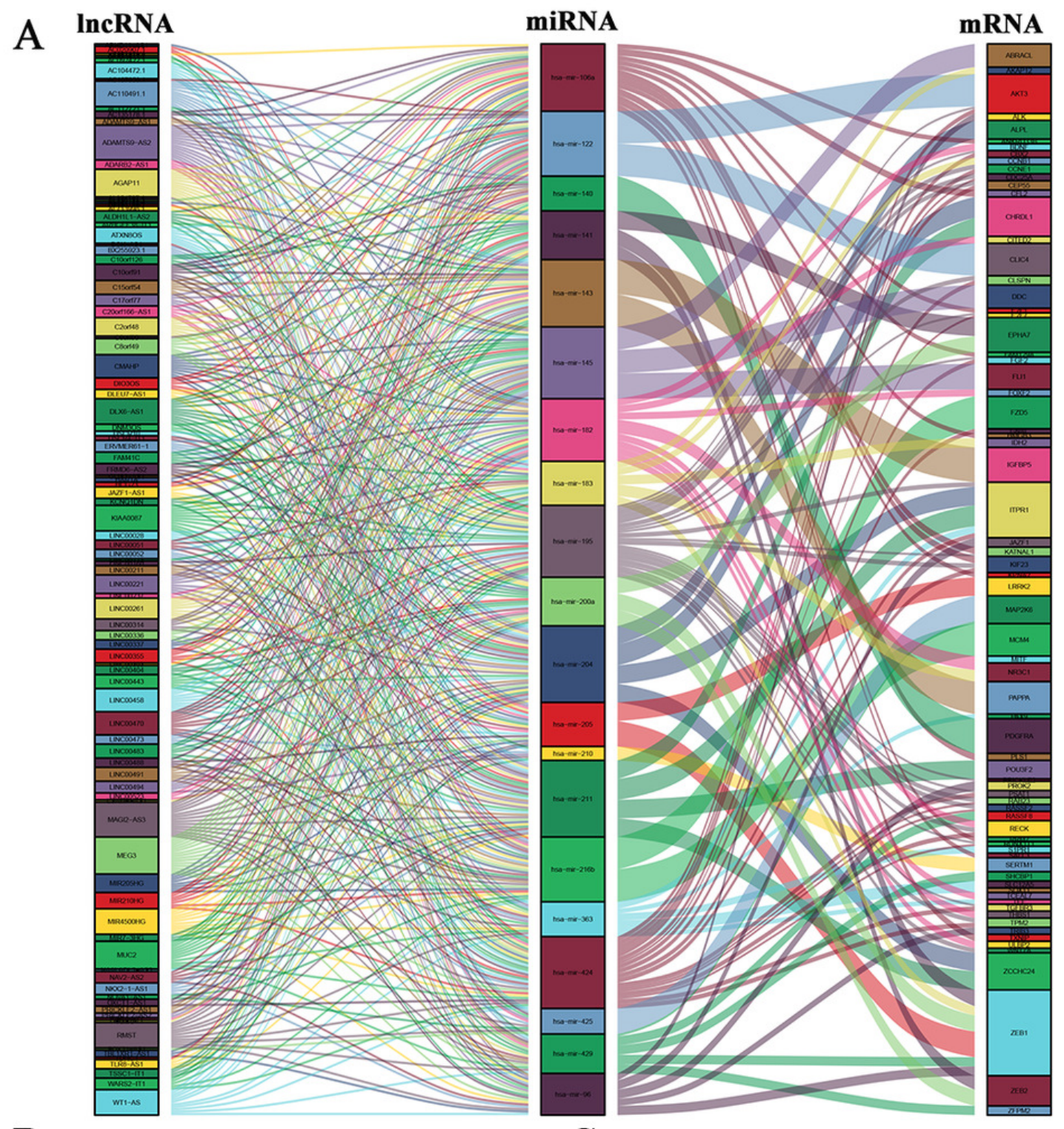

B

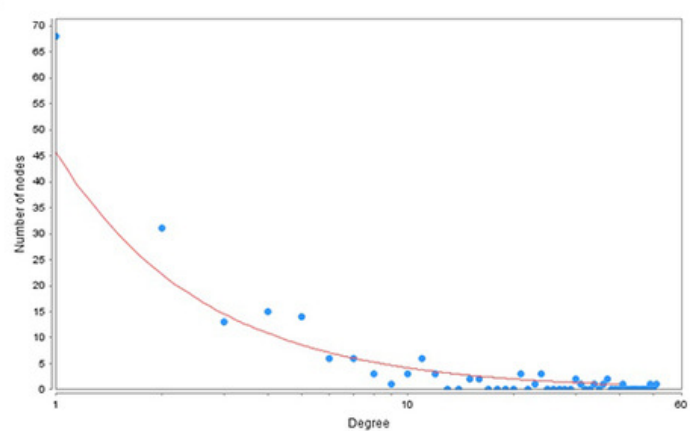

D

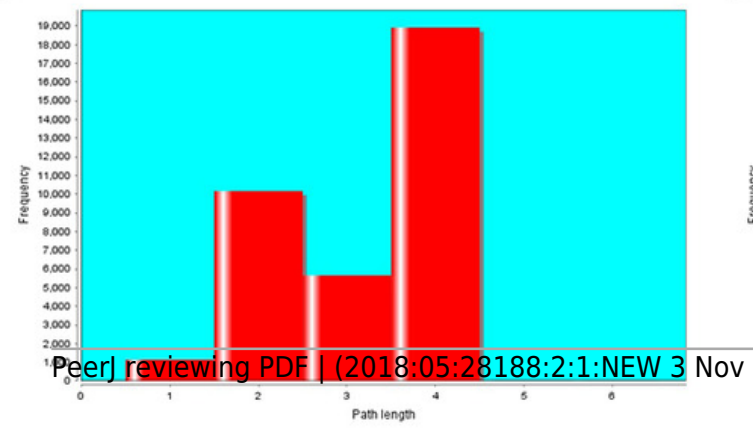

C

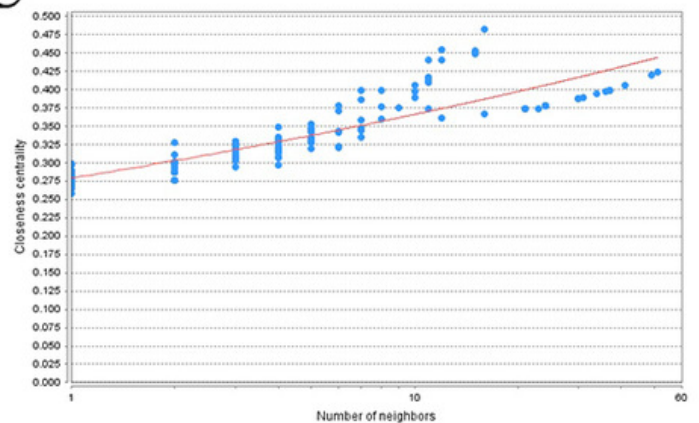

E

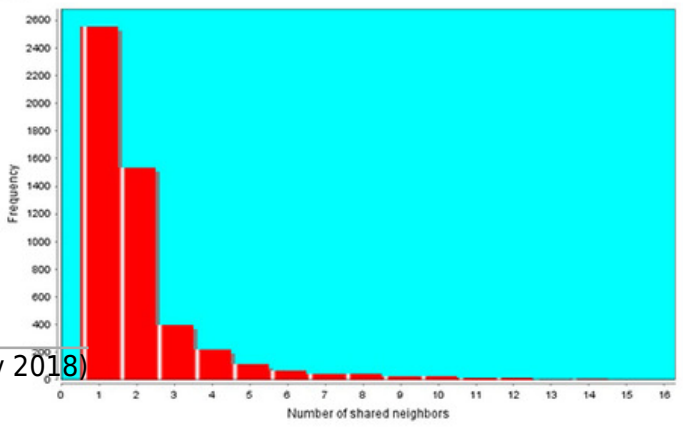




\section{Figure 3}

Regression analysis between the expression levels of DEIncRNAs and DEmRNAs targeted by hsa-mir-195

(A) Regression analysis between the expression levels of C2orf48 and PSAT1.(B) Regression analysis between the expression levels of C2orf48 and KIF23. (C) Regression analysis between the expression levels of C2orf48 andCEP55. (D) Regression analysis between the expression levels of C2orf48 and CDC25A. (E) Regression analysis between the expression levels of C2orf48 and CCNE1. (F) Regression analysis between the expression levels of C2orf48 and CBX2; The horizontal axis indicates mRNA expression, and the vertical axis indicates IncRNA expression. (Pearson's r: correlation coefficient.) The upper and right edges are histograms of gene expression.

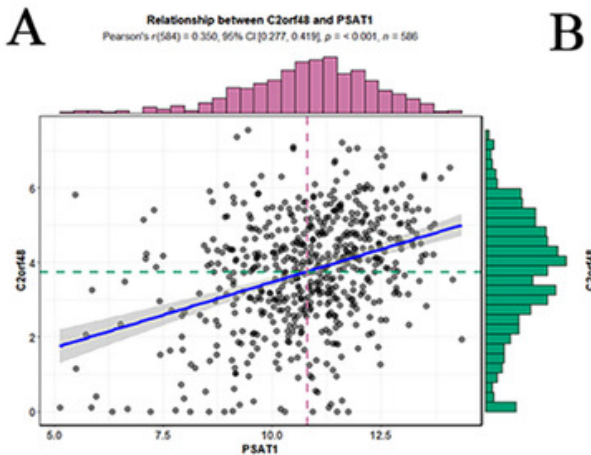

$\mathrm{D}$

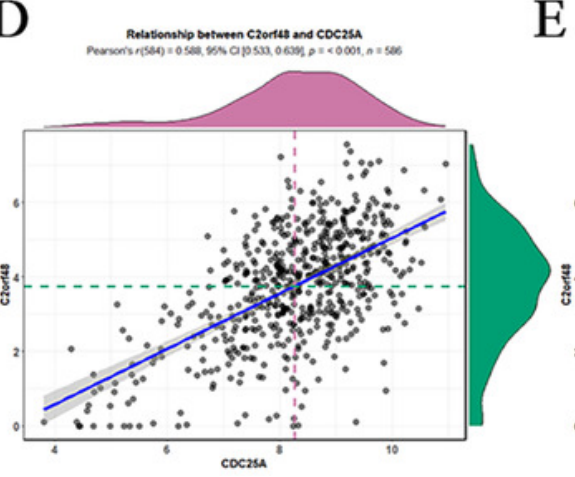

B

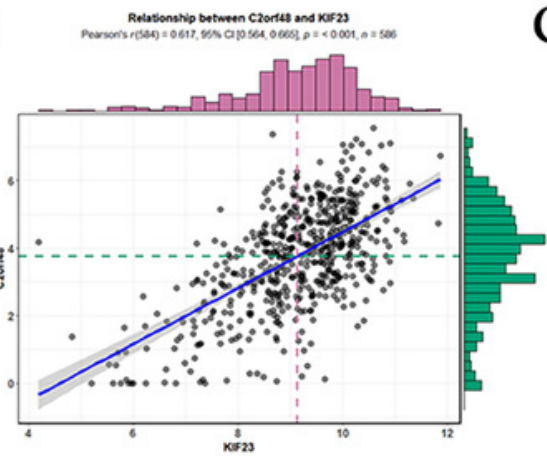

$\mathrm{E}$

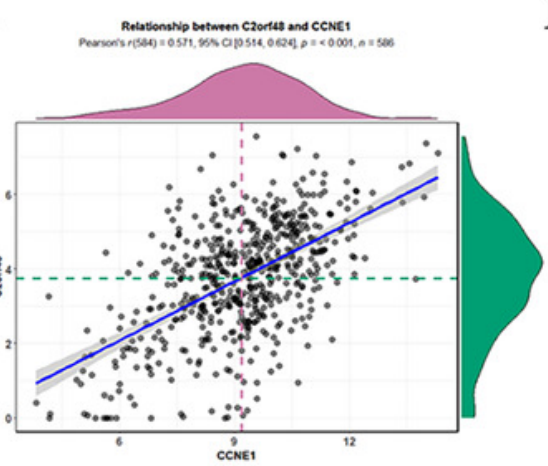

C

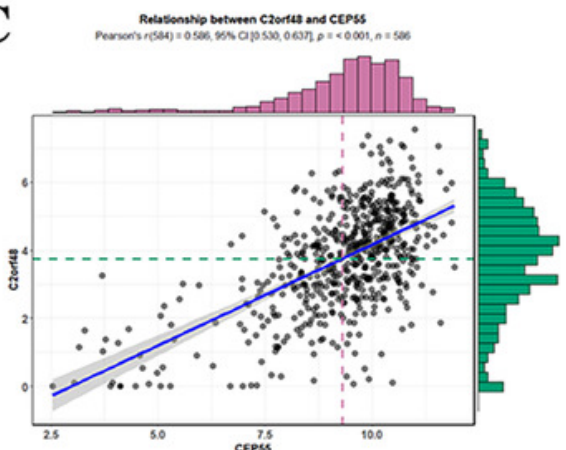

$\mathrm{F}$

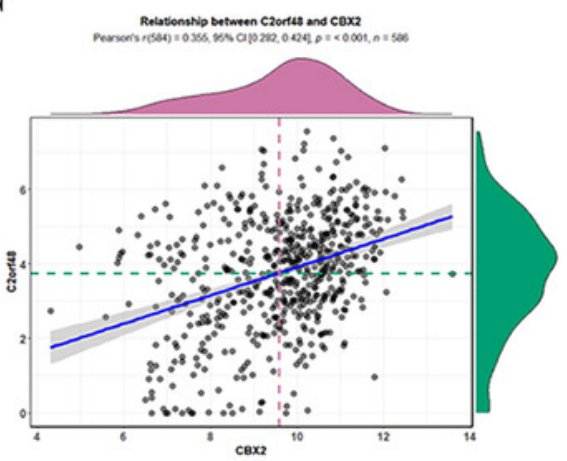




\section{Figure 4}

Biological function and pathway enrichment analysis of 73 DEmRNAs.

(A) Chord diagram showing the top 10 enriched GO clusters for 73 DEmRNAs. In each chord diagram, enriched GO clusters are shown on the right and genes contributing to enrichment are shown on the left. Upregulated DEmRNAs are displayed in red and downregulated DEmRNAs are displayed in blue. Each GO term is represented by one colored line. (B) Significant pathway enrichment of DEmRNAs. Diamonds represent the signaling pathways and ellipses represents the regulated genes.

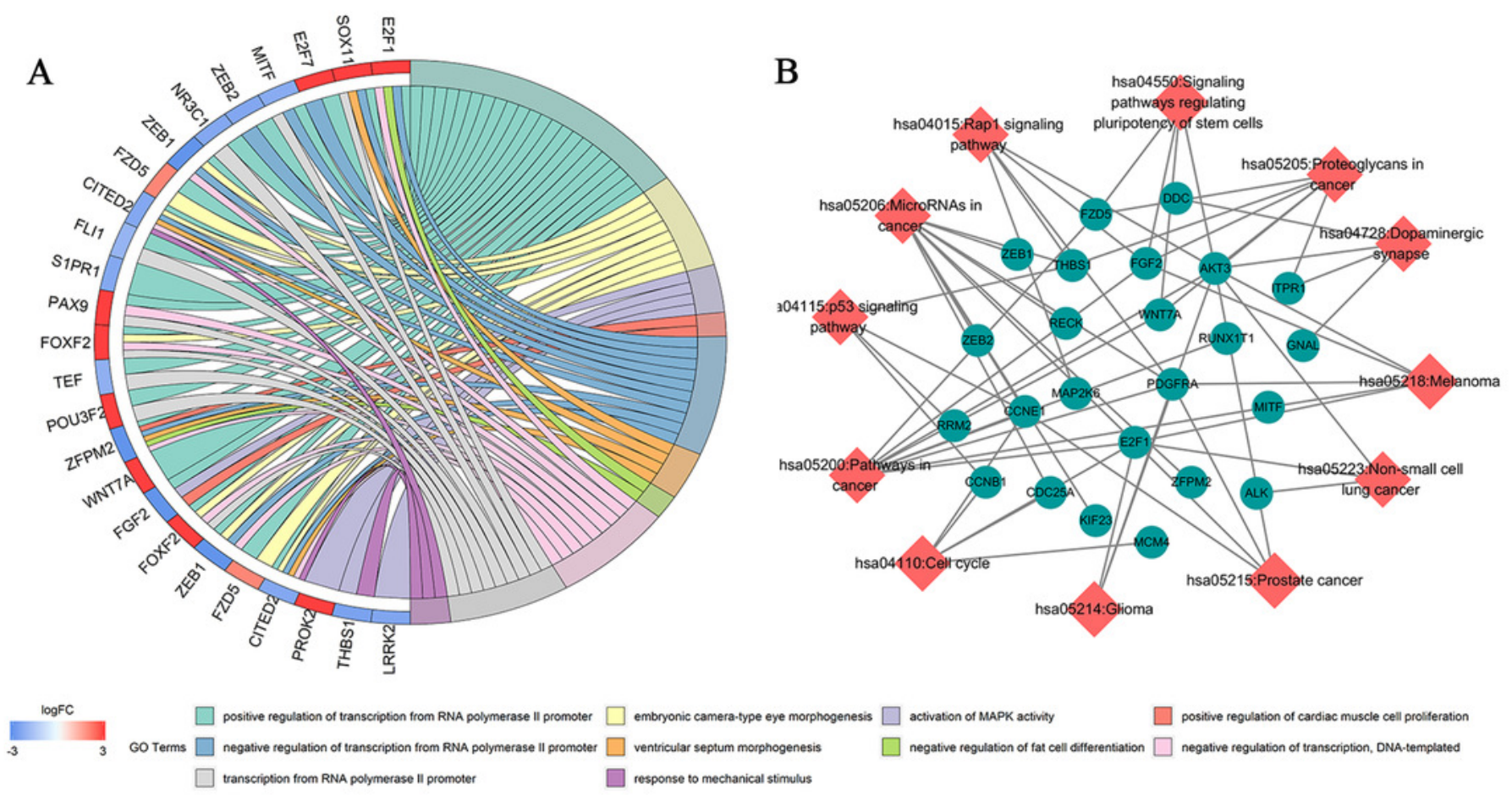




\section{Figure 5}

Kaplan-Meier survival curves for the top four IncRNAs, miRNAs and mRNAs related to overall survival and expression validation.

(A-D) Kaplan-Meier survival curves for the top four IncRNAs. (E-H)Kaplan-Meier survival curves for the top four miRNAs.(I-L)Kaplan-Meier survival curves for the top four mRNAs.(M-P) Expression validation of the top four DEmRNAs correlated with survival.(Q-T) Expression validation of the top four DEmiRNAs correlated with survival. 

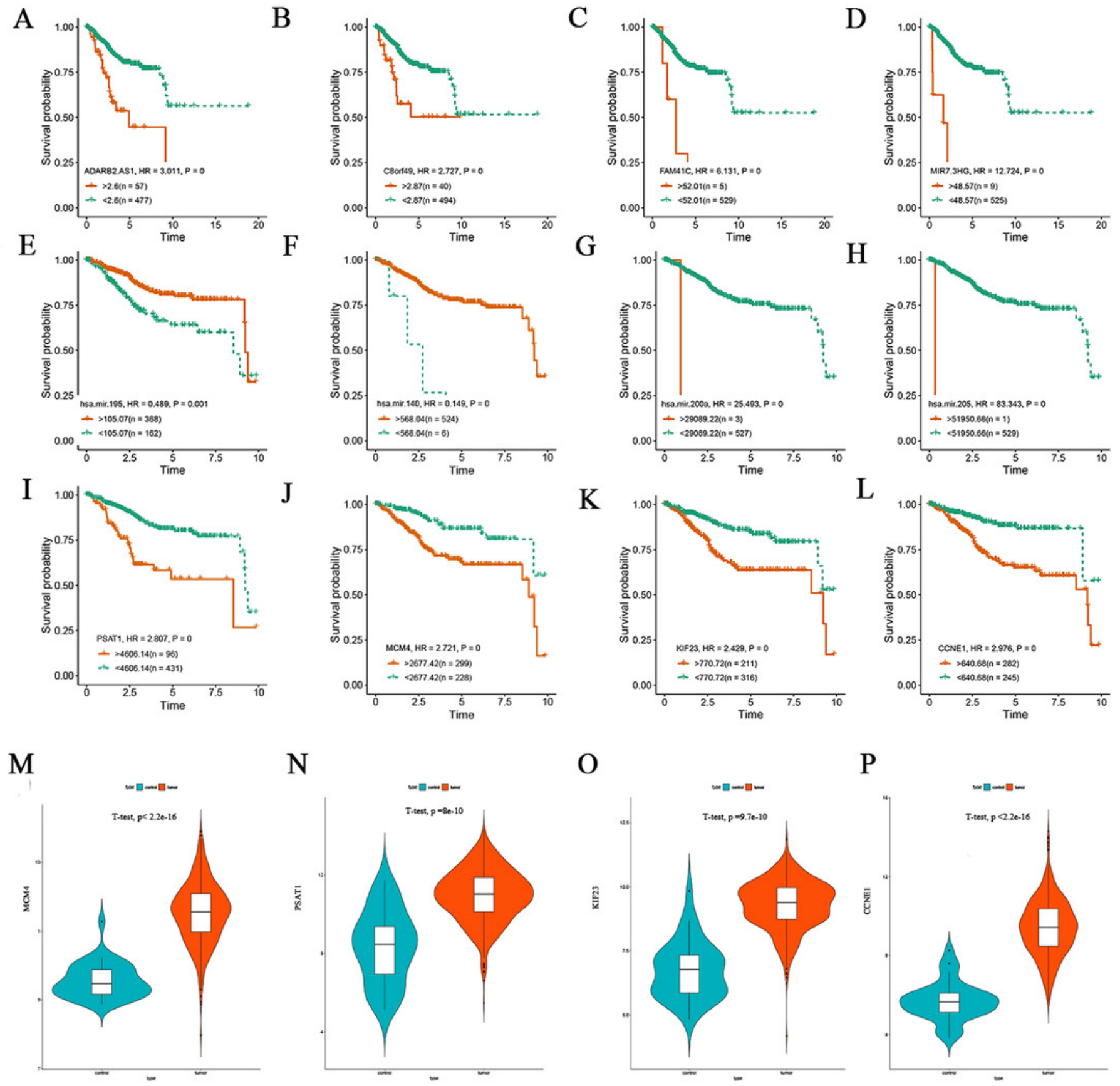

O
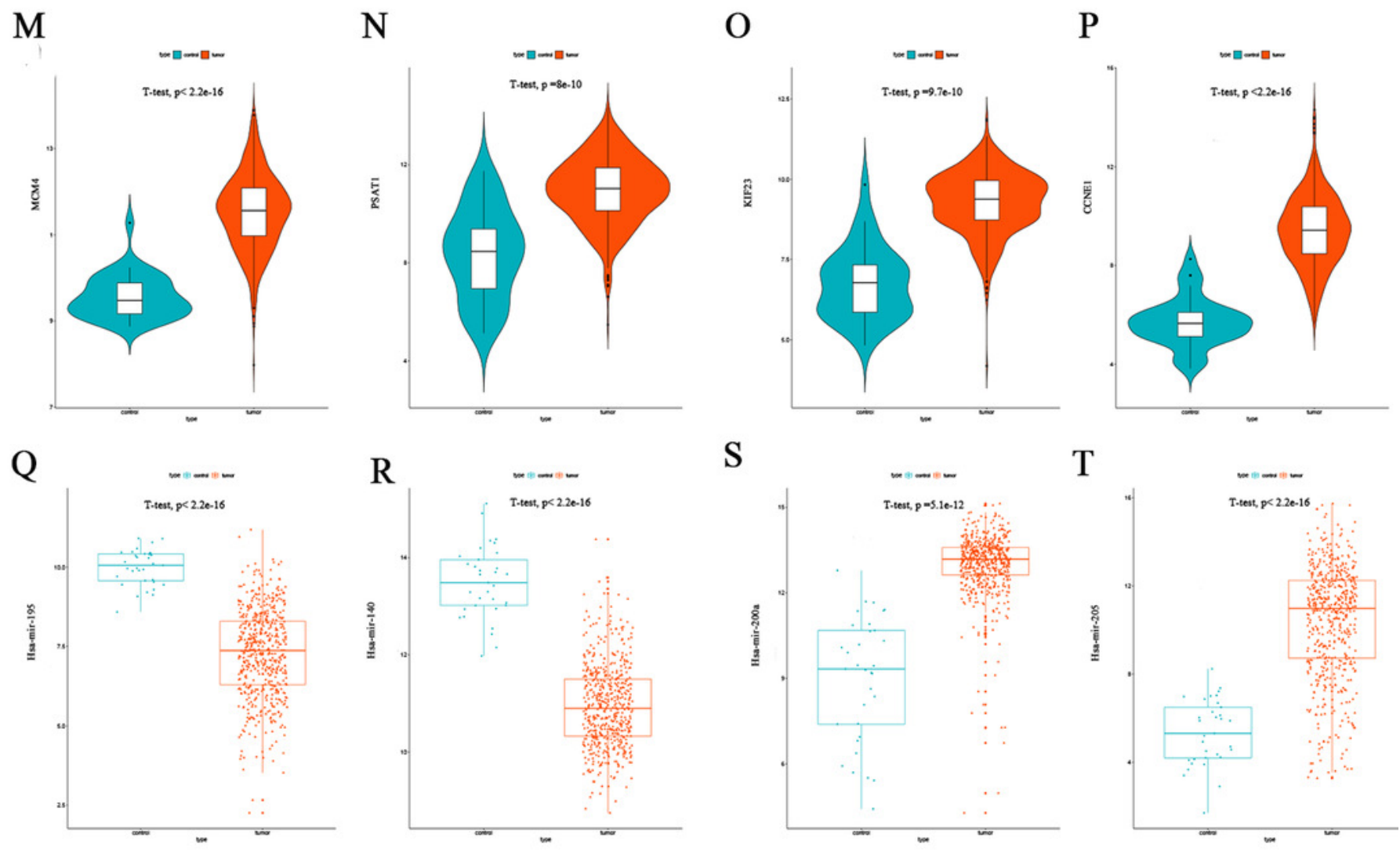

S

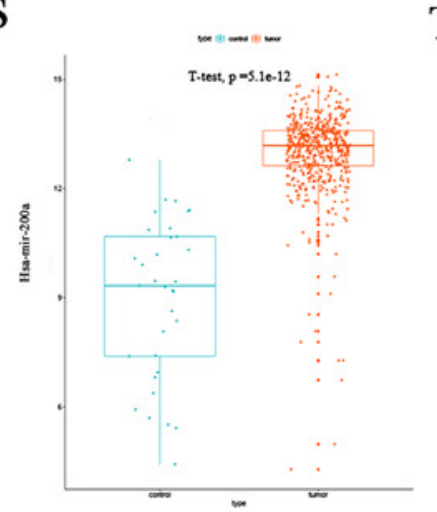

$\mathrm{T}$

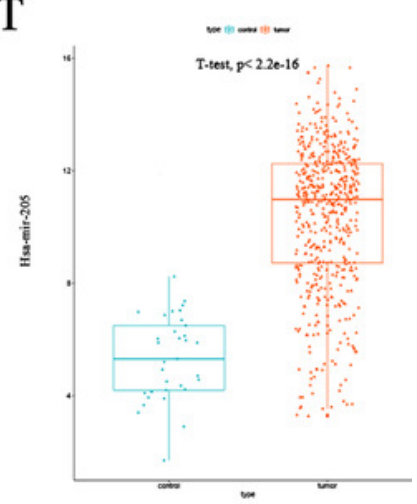




\section{Figure 6}

Correlation between gene expression and clinicopathological parameters.

(A-B) Correlations between PSAT gene expression levels and clinical stage and histological classification in the TCGA database.( C-D) Correlations between KIF23 gene expression levels and clinical stage and histological classification.(E-F) Correlations between CCNE1 gene expression levels and clinical stage and histological classification.(G-H) Correlations between MCM4 gene expression levels and clinical stage and histological classification. ***Pvalue $<0.01$. 
A

Expression of PSAT1 in UCEC based on individual cancer

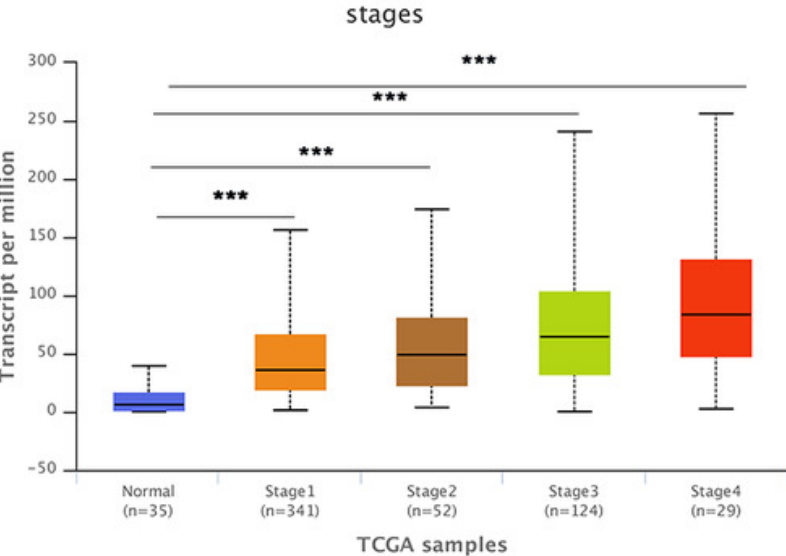

C

Expression of KIF23 in UCEC based on individual cancer stages

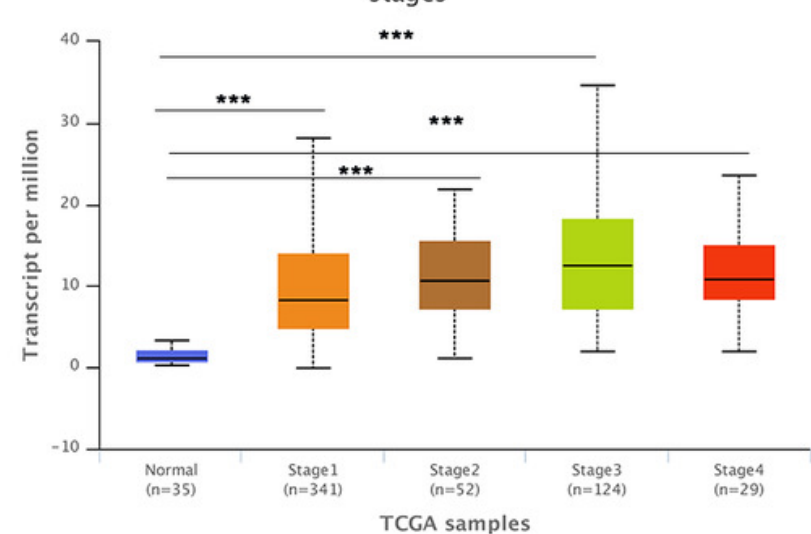

E

Expression of CCNE1 in UCEC based on individual cancer

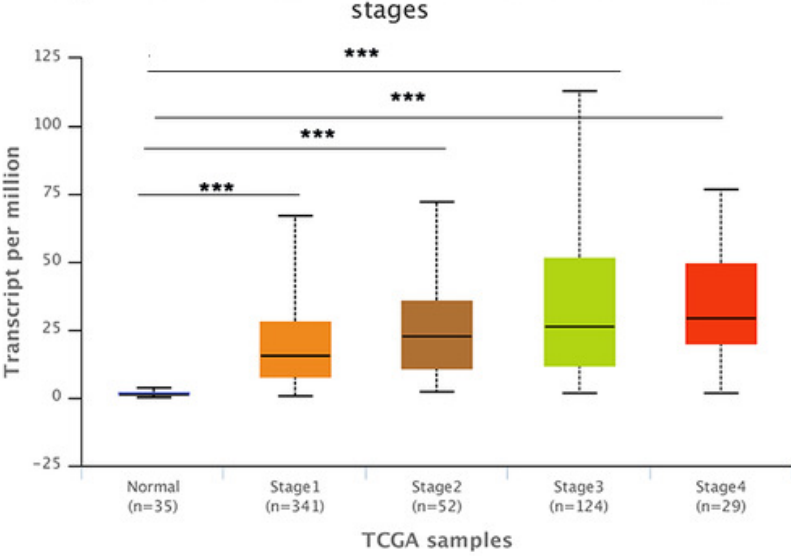

G

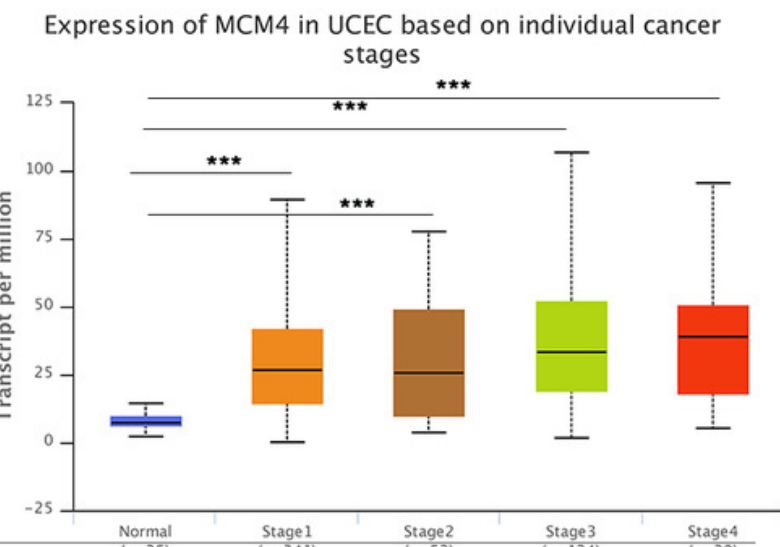

Peer] reviewining PDF |'(2018:05:28188:2:1:NEW'3 Nov 2018)
B

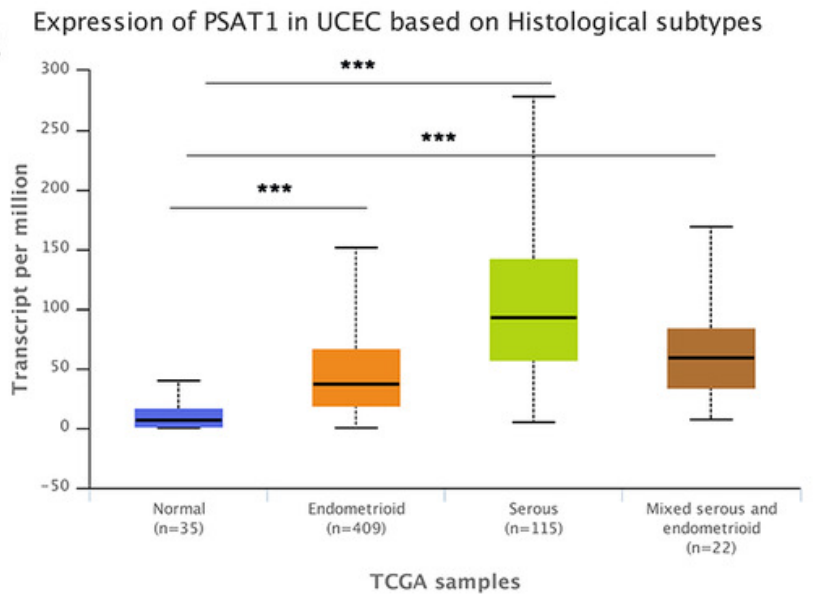

D Expression of KIF23 in UCEC based on Histological subtypes

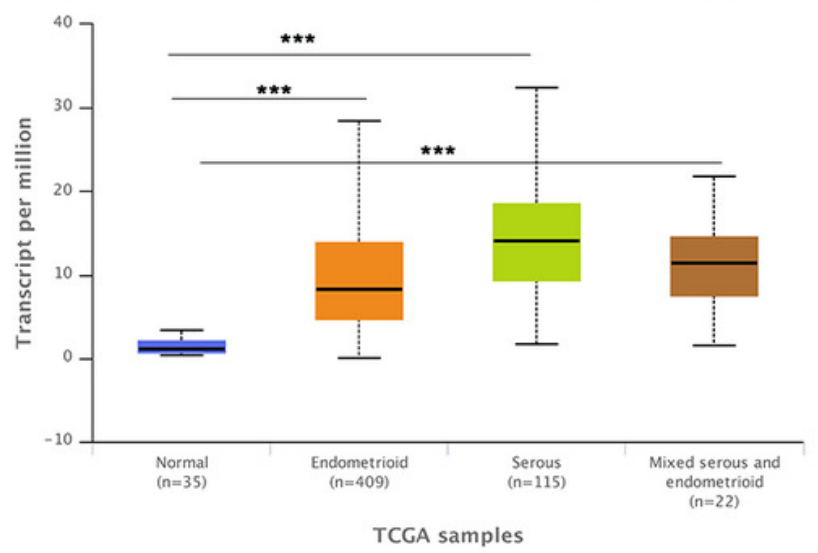

$\mathrm{F}$

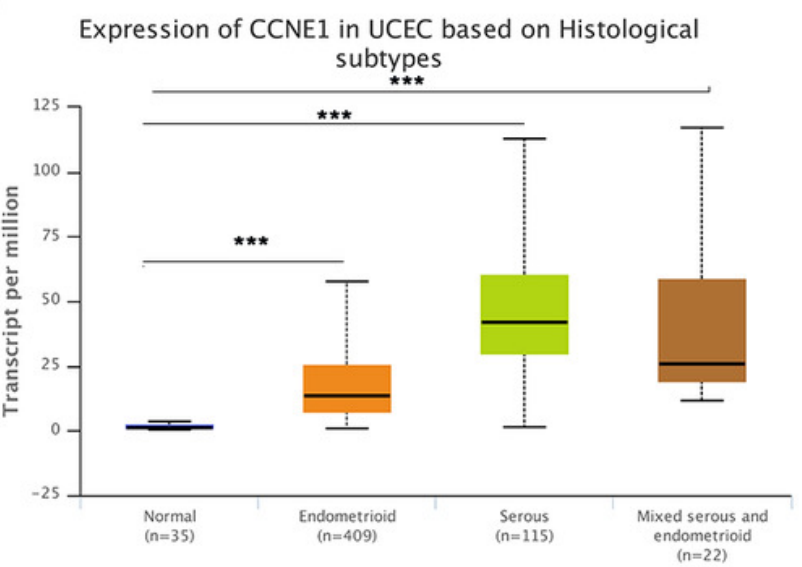

$\mathrm{H}$

TCGA samples

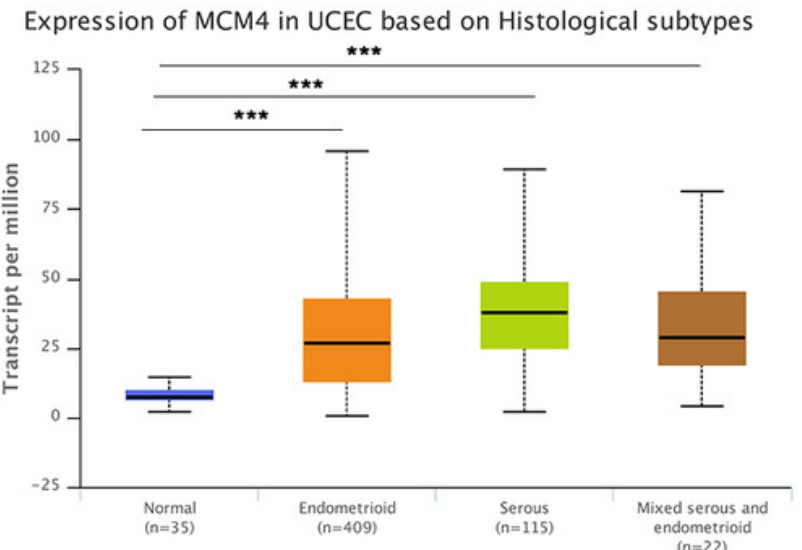




\section{Figure 7}

Validation of the top four DEmRNAs and DEmiRNAs correlated with survival.

(A) Violin plots of PSAT1mRNA expression levels in the GSE17025 validation dataset. (B)

Violin plots of KIF23 mRNA expression levels in the GSE17025 validation dataset. (C) Violin plots of MCM4 mRNA expression levels in the GSE17025 validation dataset. (D) Violin plots of CCNE1 mRNA expression levels in the GSE17025 validation dataset. (E) Scatter plots of the has-mir-195 miRNA expression levels in the GSE35794 validation dataset. (F) Scatter plots of the has-mir-140 miRNA expression levels in the GSE35794 validation dataset. (G) Scatter plots of the has-mir-200a miRNA expression levels in the GSE35794 validation dataset. $(\mathrm{H})$ Scatter plots of the has-mir-205 miRNA expression levels in the GSE35794 validation dataset.

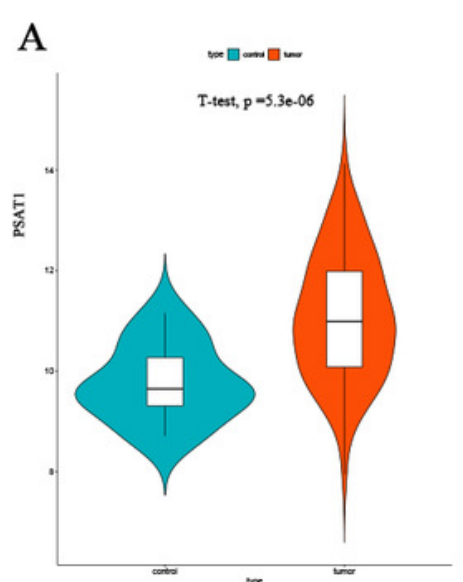

$\mathrm{E}$
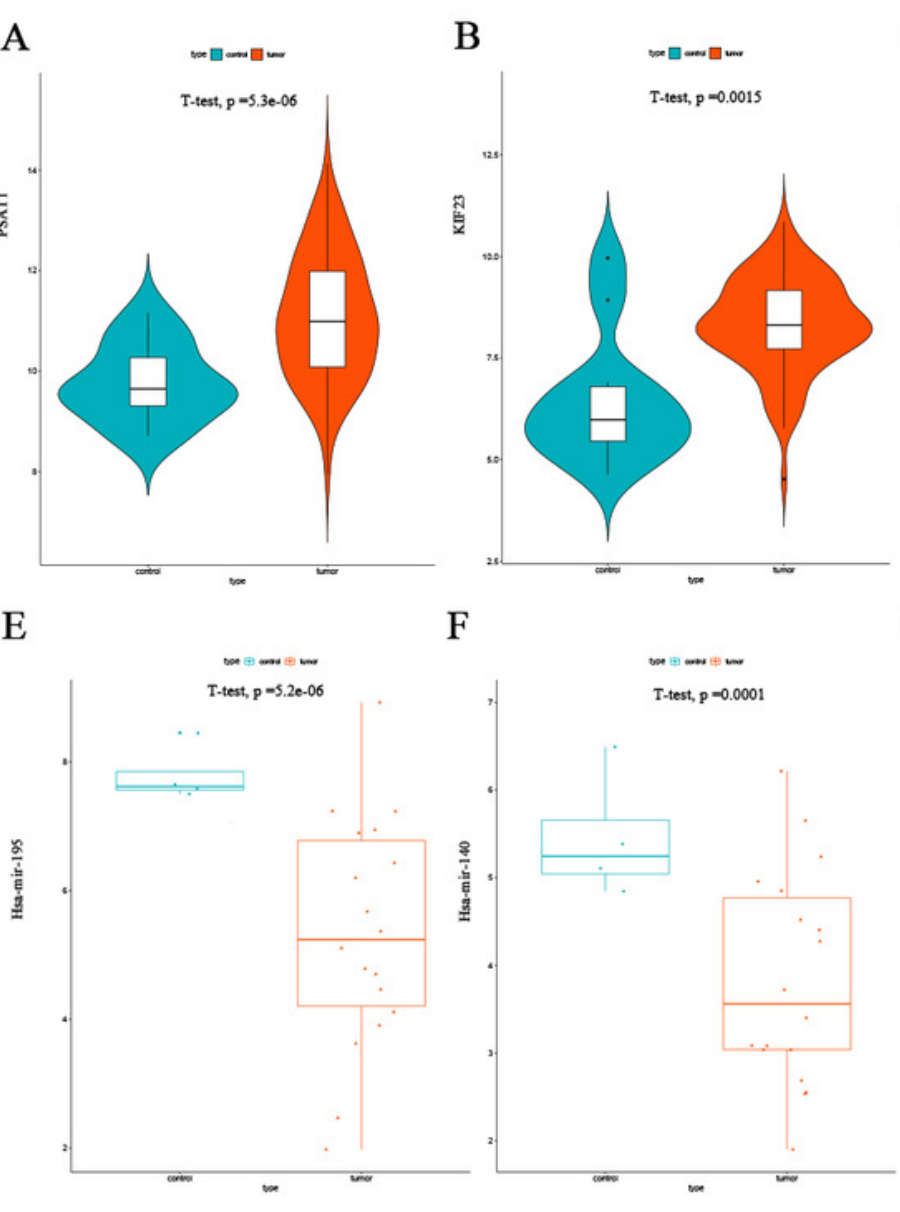

$\mathrm{F}$

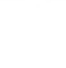

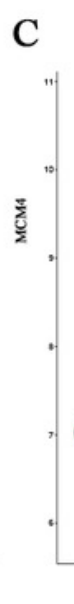

G

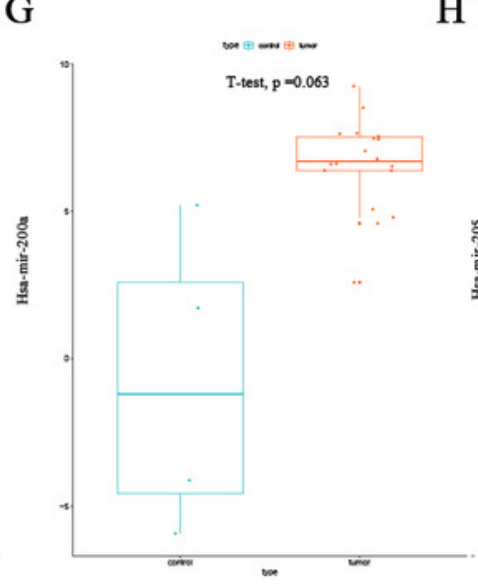

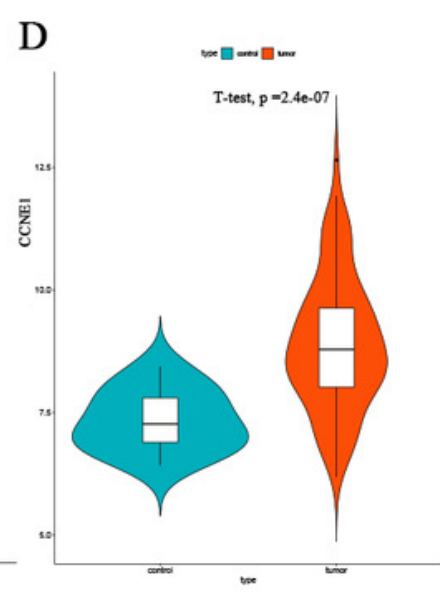

H

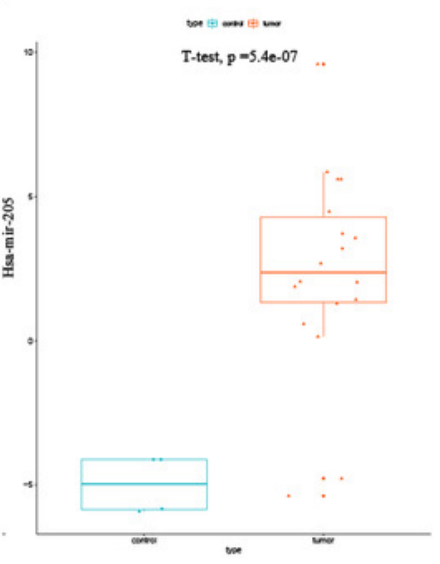




\section{Figure 8}

Flow chart of construction and analysis of ceRNA network

The flow chart of construction of IncRNAs-miRNAs-mRNA regulation network in endometrial carcinoma. 


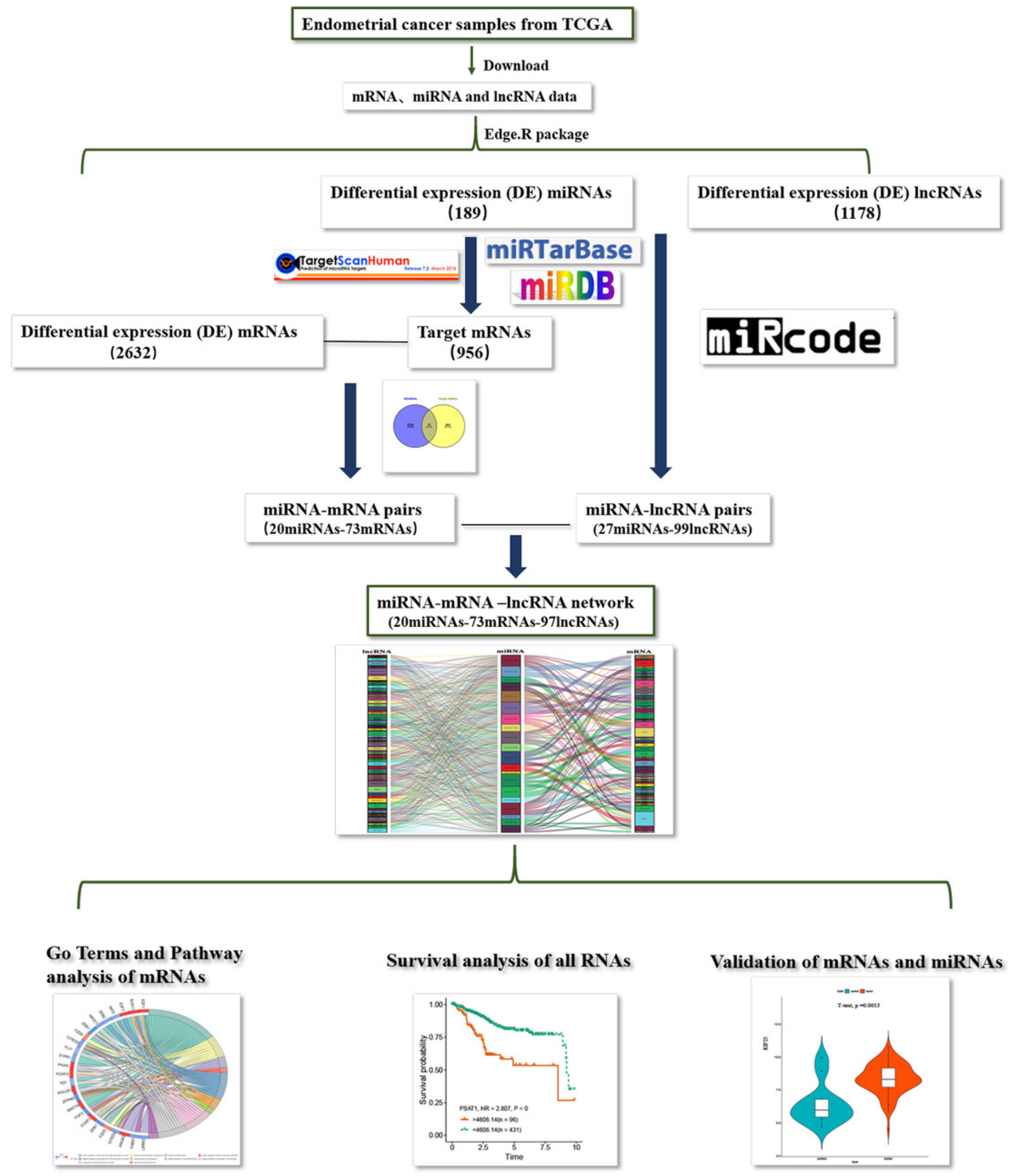

